\title{
A Model for the Detailed Analysis of Radio Links Involving Tree Canopies
}

\author{
Fernando PEREZ-FONTAN ${ }^{1}$, Milan KVICERA ${ }^{2}$, Pavel PECHAC ${ }^{2}$, Jonathan ISRAEL ${ }^{3}$ \\ ${ }^{1}$ Dept. of Signal Theory and Comm., University of Vigo, Spain \\ ${ }^{2}$ Dept. of Electromagnetic Field, Faculty of Electrical Engineering, Czech Technical University in Prague, Czech Republic \\ ${ }^{3}$ ONERA. The French Aerospace Lab, Toulouse, France
}

fpfontan@tsc.uvigo.es, \{kvicemil, pechac\}@fel.cvut.cz, Jonathan.Israel@onera.fr

Manuscript received October 24, 2016

\begin{abstract}
Detailed analysis of tree canopy interaction with incident radiowaves has mainly been limited to remote sensing for the purpose of forest classification among many other applications. This represents a monostatic configuration, unlike the case of communication links, which are bistatic. In general, link analyses have been limited to the application of simple, empirical formulas based on the use of specific attenuation values in $\mathrm{dB} / \mathrm{m}$ and the traversed vegetated mass as, e.g., the model in Recommendation ITU-R P.833-8 [1].
\end{abstract}

In remote sensing, two main techniques are used: Multiple Scattering Theory (MST) [2-5] and Radiative Transfer Theory (RT), [5], [6]. We have paid attention in the past to MST [7-10]. It was shown that a full application of MST leads to very long computation times which are unacceptable in the case where we have to analyze a scenario with several trees.

Extensive work using MST has been also presented by others in [11-16] showing the interest in this technique.

We have proposed a simplified model for scattering from tree canopies based on a hybridization of MST and a modified physical optics (PO) approach [16]. We assume that propagation through a canopy is accounted for by using the complex valued propagation constant obtained by MST. Unlike the case when the full MST is applied, the proposed approach offers significant benefits including a direct software implementation and acceptable computation times even for high frequencies and electrically large canopies. The proposed model thus replaces the coherent component in MST, significant in the forward direction, but keeps the incoherent or diffuse scattering component present in all directions. The incoherent component can be calculated within reasonable times.

Here, we present tests of the proposed model against MST using an artificial single-tree scenario at $2 \mathrm{GHz}$ and $10 \mathrm{GHz}$.

\section{Keywords}

Radiowave propagation, vegetation, scattering, multiple scattering theory

\section{Introduction}

Multiple scattering theory (MST) [1-4], computes the coherent and incoherent fields scattered by an ensemble of randomly oriented and positioned particles. Their density is low and their sizes are smaller than their separations [5]. MST has been frequently used in parallel to the Radiative Transfer Theory (RT) [5] in remote sensing, e.g. [6], to model vegetated layer scattering.

In [18-20], the scattering behavior for individual thin dielectric cylinders representing tree branches and individual flat cylinders (discs) representing leafs was presented. The scattering amplitude tensors of these tree canopy elements are used for calculating the overall scattering behavior of the whole canopy.

The applicability of MST to such scenarios has been validated in [7-16]. However, there is a significant drawback of MST preventing its widespread usage which is the very long computation times required.

As indicated, MST provides two types of fields, coherent and incoherent [5]. In the forward direction, the coherent component dominates while it practically disappears in other directions. In contrast, the incoherent component is always present with low levels.

As for shadowing behind a canopy, physical optics (PO), e.g. [21] and [22], offers a computationally efficient alternative for calculating the coherent field behind an electrically large dielectric object. It misses, however, the contributions through the canopy itself.

We will be presenting how to overcome this by taking into account the complex valued propagation constant derived with MST for the path through the canopy which adds coherently with the path around the canopy due to diffraction obtained through PO [21].

One further advantage is that we can calculate the coherently scattered fields in the near field region, which is not possible with MST where only the far fields are calculated. In this way, we are able to take into consideration the fields going through and around the canopy giving rise to smooth transitions between the line of sight (LOS) and 
shadowed sectors behind the canopy. This is in contrast with the results we would obtain if only the specific attenuation was employed, which would give rise to sharp edge effects at the transition region from LOS to non-LOS.

We carried out extensive tests where we show that the model provides results in agreement with both MST and experimental data [7-10].

\section{Multiple Scattering Theory}

The following brief description of the MST principles is based on [11], while the behavior of the individual elementary components of a canopy has been derived from [17-19].

MST assumes an incident plane wave $E_{\mathrm{i}}\left(\mathbf{r}^{\prime}\right)$ with amplitude $E_{0}$ and polarization $\mathbf{q}$ traveling in the $\mathbf{i}$ direction (Fig. 1)

$$
E_{\mathrm{i}}\left(\mathbf{r}^{\prime}\right)=\mathbf{q} E_{0} \exp \left(-j k_{0}\left(\mathbf{i} \cdot \mathbf{r}^{\prime}\right)\right)
$$

where $k_{0}$ is the propagation constant in free space. The coherent field at point $\mathbf{r}^{\prime}$ inside the canopy is

$$
E_{\text {coh }}^{\text {in }}\left(\mathbf{r}^{\prime}\right)=E_{\mathrm{i}}\left(\mathbf{r}^{\prime}\right) \exp \left\{-j\left(K-k_{0}\right) s_{1}\left(\mathbf{r}^{\prime}\right)\right\}
$$

where we introduce parameter $K$ which is the so-called effective propagation constant, which is complex valued and given by

$$
K=K^{\prime}-j K^{\prime \prime}=k_{0}+\frac{2 \pi}{k_{0}} F^{\mathrm{eq}}(\mathbf{s}, \mathbf{i})
$$

Parameter $s_{1}\left(\mathbf{r}^{\prime}\right)$ is the distance through the canopy to point $\mathbf{r}^{\prime}$ along direction i. $F^{\mathrm{eq}}(\mathbf{s}, \mathbf{i})$ is the canopy's equivalent



Fig. 1. Geometry conventions used in the tree canopy scattering model.

\begin{tabular}{|c|c|c|c|}
\hline Scatterer & $\begin{array}{c}\text { Radius } \\
(\mathbf{c m})\end{array}$ & $\begin{array}{c}\text { Length/Thickness } \\
(\mathbf{c m})\end{array}$ & $\begin{array}{c}\text { Number } \\
\text { Density }\left(\mathbf{m}^{-\mathbf{3}}\right)\end{array}$ \\
\hline Branch category 1 & 11.4 & 131 & 0.013 \\
\hline Branch category 2 & 6.0 & 99 & 0.073 \\
\hline Branch category 3 & 2.8 & 82 & 0.41 \\
\hline Branch category 4 & 0.7 & 54 & 5.1 \\
\hline Branch category 5 & 0.2 & 12 & 56 \\
\hline Leaf & 3.7 & 0.02 & 420 \\
\hline
\end{tabular}

Tab. 1. Sizes of branches and leaves [11]. scattering amplitude per unit volume in the scattering direction $\mathbf{s}$. The specific attenuation, $\alpha_{\mathrm{c}}$ in $\mathrm{dB} / \mathrm{m}$, is [11]

$$
\alpha_{c}=8.686 K^{\prime \prime} \text {. }
$$

The scattered field $E_{\mathrm{s}}(\mathbf{r})$ from any point $\mathbf{r}^{\prime}$ inside the canopy to a point $\mathbf{r}$ outside can be expressed as

$$
\left\langle E_{\mathrm{s}}(\mathbf{r})\right\rangle=\int_{V} F^{\mathrm{eq}}(\mathbf{s}, \mathbf{i}) \frac{\exp \left(-j k_{0}\left|\mathbf{r}-\mathbf{r}^{\prime}\right|\right)}{\left|\mathbf{r}-\mathbf{r}^{\prime}\right|} E_{\text {coh }}^{\text {in }}\left(\mathbf{r}^{\prime}\right) d V^{\prime} .
$$

The incoherent part is given by

$$
\left\langle\left|E_{\mathrm{s}}(\mathbf{r})\right|^{2}\right\rangle=\int_{V}\left|V\left(\mathbf{r}, \mathbf{r}^{\prime}\right)\right|^{2}\left|E_{\mathrm{coh}}^{\text {in }}\left(\mathbf{r}^{\prime}\right)\right|^{2} d V^{\prime}
$$

where \langle\rangle means statistical averaging and $V\left(\mathbf{r}, \mathbf{r}^{\prime}\right)$ is an operator given by

$$
V\left(\mathbf{r}, \mathbf{r}^{\prime}\right)=F^{\mathrm{eq}}(\mathbf{s}, \mathbf{i}) \frac{\exp \left(-j k_{0}\left|\mathbf{r}-\mathbf{r}^{\prime}\right|-j\left(K-k_{0}\right) s_{2}\left(\mathbf{r}^{\prime}\right)\right)}{\left|\mathbf{r}-\mathbf{r}^{\prime}\right|}
$$

where $s_{2}\left(\mathbf{r}^{\prime}\right)$ is the distance through the canopy from a point $\mathbf{r}^{\prime}$ to the point $\mathbf{r}$ along $\mathbf{s}$. The coherent part is a complex electric field while the incoherent component is a power, i.e., with no phase.

The canopy is assumed to consist of various branch types of different sizes and leaves. A configuration proposed in [11] and [12] uses five branch (cylinder) categories, Tab. 1. In addition, we have the leaves (discs). Their relative complex permittivity is $\varepsilon_{b}=28-7 j$ for branches, and $\varepsilon_{l}=31-8 j$ for leaves.

Their orientations are random and are described by two angles, $\alpha$ and $\beta$, with respect to the tree reference coordinate frame, as in [11] or [12]. In [11], it is assumed that uniform distribution, $f_{\Omega}(\alpha, \beta)$, adequately represents the orientations of single branches $b$ and leaves $l$. However, the orientation angle range for the larger branches tends to be limited and centered about the vertical direction.

The average scattering amplitude can be obtained by computing the mean taking into account the distribution of orientations

$$
\left\langle F^{b, l}(\mathbf{s}, \mathbf{i})\right\rangle=\int_{0}^{2 \pi} d \alpha \int_{0}^{\pi / 2} d \beta F^{b, l}(\mathbf{s}, \mathbf{i}) f_{\Omega}(\alpha, \beta)
$$

where $F^{b, l}(\mathbf{s}, \mathbf{i})$ is the scattering amplitude tensor for the branches or leaves [18-20].

Moreover, we need to take into account the respective number densities $\rho_{b, l}$. Thus, for the whole canopy, we get

$$
F^{\mathrm{eq}}(\mathbf{s}, \mathbf{i})=\sum_{b} \rho_{b} F^{b}(\mathbf{s}, \mathbf{i})+\rho_{l} F^{l}(\mathbf{s}, \mathbf{i}) \cdot
$$

Finally, we can convert scattering amplitudes to cross sections for a single branch or leaf,

$$
\sigma^{b, l}(\mathbf{s}, \mathbf{i})=4 \pi \int_{0}^{2 \pi} d \alpha \int_{0}^{\pi / 2} d \beta\left|F^{b, l}(\mathbf{s}, \mathbf{i})\right|^{2} f_{\Omega}(\alpha, \beta)
$$


and for the overall canopy in terms of an equivalent scattering cross section per unit volume in $\mathrm{m}^{2} / \mathrm{m}^{3}$ as

$$
\sigma^{\mathrm{eq}}(\mathbf{s}, \mathbf{i})=4 \pi \sum_{b} \rho_{b}\left|F^{b}(\mathbf{s}, \mathbf{i})\right|^{2}+4 \pi \rho_{l}\left|F^{l}(\mathbf{s}, \mathbf{i})\right|^{2} .
$$

The validity of the models for individual canopy elements in [18-20] corresponds to the cases where the branch lengths are sufficiently greater than their radii, and the leaves are electrically thin.

Now we provide some examples to illustrate the behavior of the model for different input parameters. First we discuss the behavior of a canopy illuminated by a vertically polarized plane wave. The tree follows the configuration in Tab. 1 [11]. The incidence is along the horizontal direction and the scattering characteristics are investigated in the horizontal $x y$ plane for scattering angles $\varphi_{\mathrm{S}}$ from $0^{\circ}$ to $180^{\circ}$ at the distance of $50 \mathrm{~m}$ from the canopy center.

Figure 2 illustrates the effect of the frequency in the scattering amplitudes for branches of category one and leaves at $5 \mathrm{GHz}$ and $10 \mathrm{GHz}$ together with the resulting overall equivalent scattering amplitudes $F^{\mathrm{eq}}(\mathbf{s}, \mathbf{i})$.

The problem with the model is that very fine canopy volume integration in (5) and (6) is required, mainly for the coherent component. The incoherent component is not so sensitive and larger elementary volumes can be used, thus

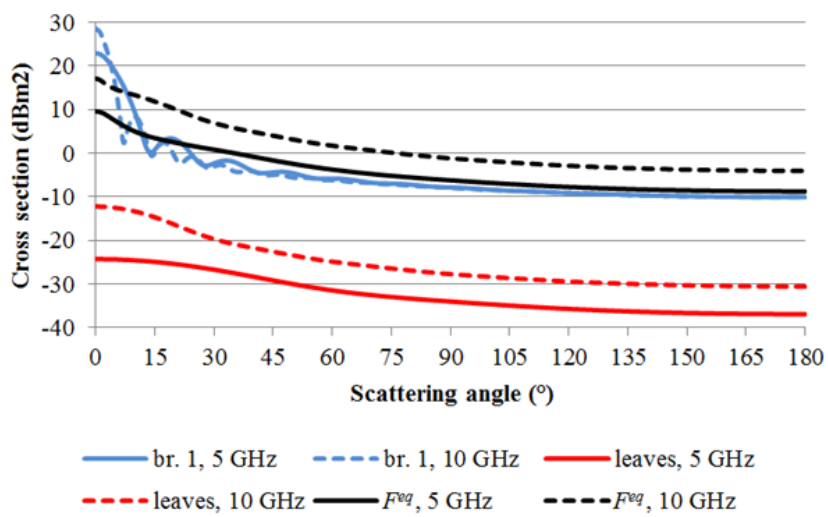

Fig. 2. Scattering amplitudes for branches of category one and leaves at $5 \mathrm{GHz}$ and $10 \mathrm{GHz}$ and overall resulting equivalent scattering amplitude.

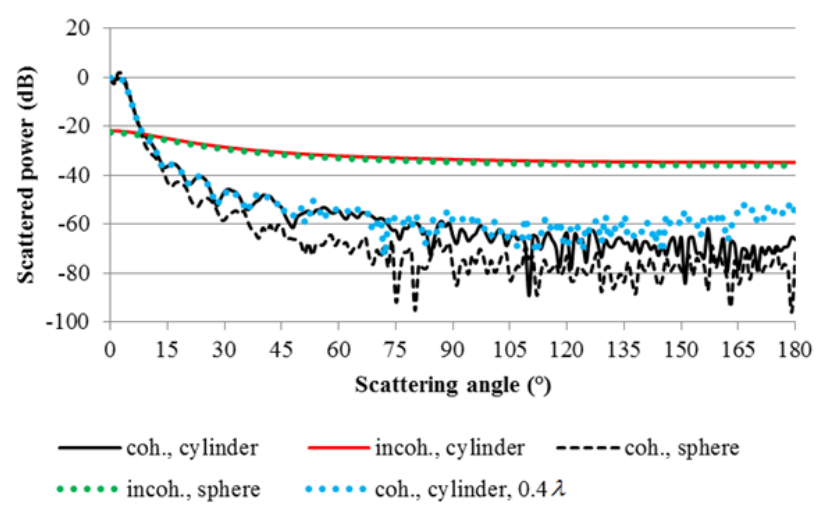

Fig. 3. Coherent and incoherent scattered fields at $2 \mathrm{GHz}$ for a tree canopy: cylindrical (radius of $5 \mathrm{~m}$, height $8 \mathrm{~m}$ ) and spherical (radius of $5 \mathrm{~m}$ ). reducing the required computation time. Typically, for the coherent component, $d V^{\prime}$ must be set to cubes of side smaller than $0.1 \lambda$ which for large canopies and high frequencies results in very long computation times. This is the main shortcoming of MST which prevents us from using it directly in large propagation scenarios.

To illustrate the results of the overall model in Fig. 3, we present the obtained coherent and incoherent contributions at $2 \mathrm{GHz}$ for cylindrical and spherical canopies of approximately the same volume. We can see how the coherent contributions dominate only in the forward direction and then fall off sharply to be overcome by the incoherent component.

As for the specific attenuation for this configuration, it can be determined from the imaginary part of $K$ as $0.97 \mathrm{~dB} / \mathrm{m}$ (4), well in line with empirical results provided in [1] or [23-25].

\section{Physical Optics Approximation}

In accordance with [21] and [22], we consider both the electric $\mathbf{J}_{\mathrm{eq}}$ and magnetic $\mathbf{M}_{\mathrm{eq}}$ equivalent surface currents in our implementation.

We can start off by assuming a perfectly absorbing body, that is, where no reflected fields are generated. Based on this assumption, the following relations apply [20], [21]

$$
\begin{gathered}
\mathbf{M}_{\mathrm{eq}}=-\mathbf{n} \times \mathbf{E}_{\mathrm{inc}}, \\
\mathbf{J}_{\mathrm{eq}}=\mathbf{n} \times \mathbf{H}_{\mathrm{inc}}
\end{gathered}
$$

where $\mathbf{n}$ is the surface's outward pointing unit normal vector at $\mathbf{r}^{\prime}$. The surface samples must be illuminated by the incident plane wave, i.e. $\mathbf{k}_{\text {inc }} \mathbf{n}<0$.

The electric and magnetic scattered near fields at point $\mathbf{r}$ are [21]

$$
\begin{aligned}
& \mathbf{H}_{\text {scat }, \mathrm{J}}=-j k_{0} \int \frac{\exp \left(-j k_{0}\left|\mathbf{r}-\mathbf{r}^{\prime}\right|\right)}{4 \pi\left|\mathbf{r}-\mathbf{r}^{\prime}\right|} \frac{\mathbf{r}-\mathbf{r}^{\prime}}{\left|\mathbf{r}-\mathbf{r}^{\prime}\right|} \times \mathbf{J}_{\mathrm{eq}} d S^{\prime}, \\
& \mathbf{E}_{\text {scat }, \mathrm{M}}=j k_{0} \int \frac{\exp \left(-j k_{0}\left|\mathbf{r}-\mathbf{r}^{\prime}\right|\right)}{4 \pi\left|\mathbf{r}-\mathbf{r}^{\prime}\right|} \frac{\mathbf{r}-\mathbf{r}^{\prime}}{\left|\mathbf{r}-\mathbf{r}^{\prime}\right|} \times \mathbf{M}_{\mathrm{eq}} d S^{\prime}
\end{aligned}
$$

where $d S^{\prime}$ is the surface discretization element. From these two fields, their associated fields, $\mathbf{E}_{\text {scat }, \mathrm{J}}$ and $\mathbf{H}_{\text {scat }, \mathrm{M}}$, are computed.

The blockage of the original plane wave fields, $\mathbf{E}_{\mathrm{dir}}$ and $\mathbf{H}_{\text {dir }}$, is introduced by the scattered fields [21]. When added together, the resulting fields in the presence of a perfectly absorbing obstacle are

$$
\begin{gathered}
\mathbf{E}_{\mathrm{B}}=\mathbf{E}_{\text {dir }}+\left(\mathbf{E}_{\text {scat }, \mathrm{J}}+\mathbf{E}_{\text {scat }, \mathrm{M}}\right)=\mathbf{E}_{\text {dir }}+\mathbf{E}_{\text {block }}, \\
\mathbf{H}_{\mathrm{B}}=\mathbf{H}_{\text {dir }}+\left(\mathbf{H}_{\text {scat }, \mathrm{J}}+\mathbf{H}_{\text {scat }, \mathrm{M}}\right)=\mathbf{H}_{\text {dir }}+\mathbf{H}_{\text {block }}
\end{gathered}
$$

giving rise to a diffraction pattern around the canopy. The whole approach is illustrated in Fig. 4. 


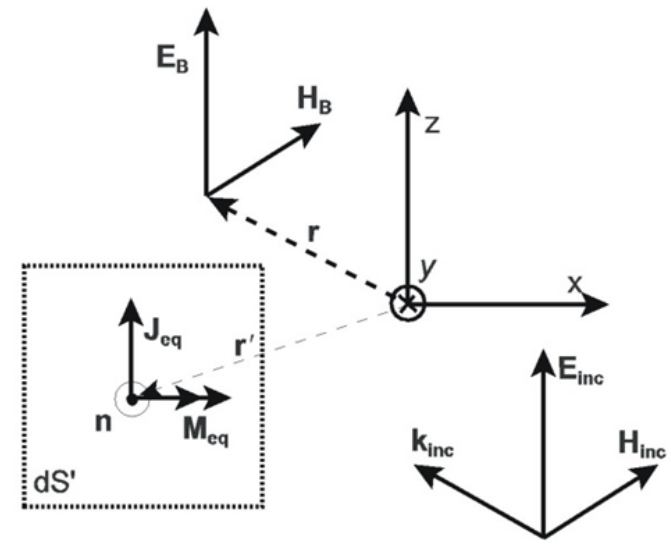

Fig. 4. Principles of Physical Optics.

\section{Simplified Model for the Coherent Component}

In the previous section, we looked into PO for the case of a completely absorbing body. We want to address the propagation path through a canopy based on $K$ and hybridize it with PO. This allows us to avoid the heavy computational load required by MST.

Based on (16), we can obtain the original fields at $\mathbf{r}$, i.e. as if no perfectly absorbing object was present, by eliminating the blockage field as

$$
\mathbf{E}_{\text {dir }}=\mathbf{E}_{\text {dir }}+\mathbf{E}_{\text {block }}-\mathbf{E}_{\text {block }} \text {. }
$$

In terms of PO, for $\mathbf{n}$ pointing in the opposite direction than in Fig. 1, $-\mathbf{E}_{\text {block }}$ can be obtained as if $d S^{\prime}$ was a radiating aperture instead of a blocking element, thus providing an aperture field $\mathbf{E}_{\text {aper }}$. This follows Babinet's principle in optics [22] stating that the field in case of no screen $\left(\mathbf{E}_{\text {dir }}\right)$ equals the sum of the field behind a screen with an opening/aperture $\left(-\mathbf{E}_{\text {block }}\right)$ and the field of the complementary structure $\left(\mathbf{E}_{\text {block }}\right)$.

In our approximated model, we keep the fields $\mathbf{E}_{\mathrm{B}}$ and $\mathbf{H}_{\mathrm{B}}$, (16) and (17), and modify $\mathbf{E}_{\text {aper }}$ and $\mathbf{H}_{\text {aper }}$ based on the complex effective propagation constant of the body. The resulting total fields are then

$$
\begin{gathered}
\mathbf{E}_{\text {tot }}=\mathbf{E}_{\mathrm{B}}+\mathbf{E}_{\text {aper }}=\left(\mathbf{E}_{\text {dir }}+\mathbf{E}_{\text {block }}\right)+\mathbf{E}_{\text {aper }}, \\
\mathbf{H}_{\text {tot }}=\mathbf{H}_{\mathrm{B}}+\mathbf{H}_{\text {aper }}=\left(\mathbf{H}_{\text {dir }}+\mathbf{H}_{\text {block }}\right)+\mathbf{H}_{\text {aper }} .
\end{gathered}
$$

In case of a completely transparent body we would have $\mathbf{E}_{\text {aper }}=-\mathbf{E}_{\text {block }}$ and (19) would predict that $\mathbf{E}_{\text {tot }}$ is equal to $\mathbf{E}_{\text {dir }}$, i.e.,

$$
\mathbf{E}_{\text {tot }}=\left(\mathbf{E}_{\text {dir }}+\mathbf{E}_{\text {block }}\right)-\mathbf{E}_{\text {block }}=\mathbf{E}_{\text {dir }}
$$

In the other extreme case, where the body is totally opaque, $\mathbf{E}_{\text {aper }}$ must be accordingly adjusted to a null value, yielding

$$
\mathbf{E}_{\text {tot }}=\left(\mathbf{E}_{\text {dir }}+\mathbf{E}_{\text {block }}\right)+0=\mathbf{E}_{\mathrm{B}} \text {. }
$$

A similar reasoning applies to the magnetic fields.

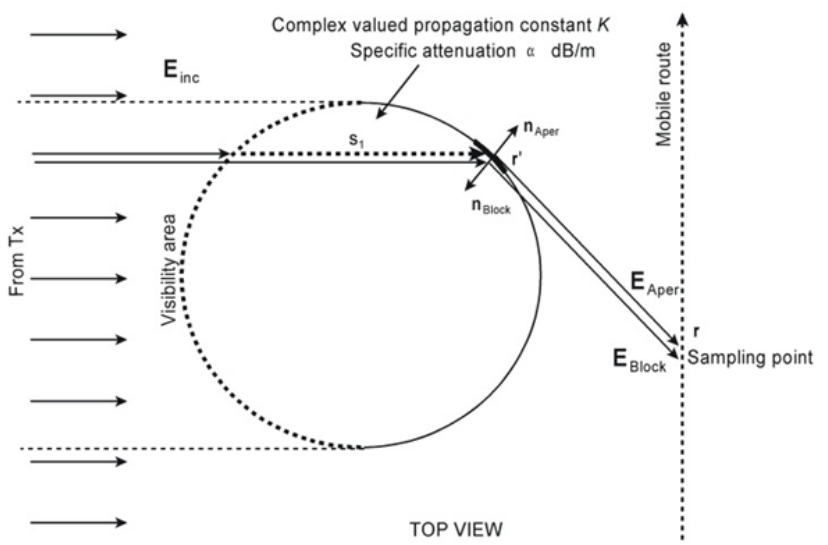

Fig. 5. Proposed hybrid model components and geometrical conventions taken.

With reference to Fig. 5, we describe our approximations in some more details.

In PO, generally, the outer surface is tested for visibility by the incoming plane wave to enable the calculation of the scattered fields. However, here, in order to reduce computation time, it is more advantageous to consider the inner surface and assume it to be illuminated ( $\mathbf{k}_{\text {inc }} \mathbf{n}>0$ for n pointing outwards the surface, solid line in Fig. 5). Then, $K$ can be applied directly to the incoming wave considering the path through the canopy in the direction of $\mathbf{k}_{\text {inc }}$ towards $\mathbf{r}^{\prime}$ as

$$
\mathbf{E}_{\mathrm{inc}}=E_{0} \exp \left(-j k_{0} \mathbf{k}_{\mathrm{inc}} \cdot \mathbf{r}^{\prime}\right) \exp \left[-j\left(K-k_{0}\right) s_{1}\left(\mathbf{r}^{\prime}\right)\right] .
$$

From this we obtain

$$
\begin{gathered}
\mathbf{E}_{\text {aper }}=\mathbf{E}_{\text {scat }, \mathrm{J}}+\mathbf{E}_{\text {scat }, \mathrm{M}}, \\
\mathbf{H}_{\text {aper }}=\mathbf{H}_{\text {scat }, \mathrm{J}}+\mathbf{H}_{\text {scat }, \mathrm{M}},
\end{gathered}
$$

with $\mathbf{n}=\mathbf{n}_{\text {aper }}$ pointing as indicated in Fig. 5. This makes the model very straightforward and with low computational load. Should we had chosen the surface visible from the incident ray (dashed line in Fig. 5), the number of computations would have been much larger.

The blockage fields, $\mathbf{E}_{\text {block }}$ and $\mathbf{H}_{\text {block}}$, are obtained with $\mathbf{n}=\mathbf{n}_{\text {block }}$ as in Fig. 2. The direct fields at $\mathbf{r}$ are

$$
\begin{aligned}
& \mathbf{E}_{\mathrm{inc}}=E_{0} \exp (-j \mathbf{k} \cdot \mathbf{r}), \\
& \mathbf{H}_{\mathrm{inc}}=\left(1 / Z_{0}\right) \mathbf{k}_{\mathrm{inc}} \times \mathbf{E}_{\mathrm{inc}} .
\end{aligned}
$$

The fields inside the canopy are computed according to [11], taking into consideration the propagation constant $K$, i.e.

$$
\mathbf{E}\left(\mathbf{r}^{\prime}\right) \sim \exp \left\{-j K s_{1}\left(\mathbf{r}^{\prime}\right)\right\}=\exp \left\{-j\left(K^{\prime}-j K^{\prime \prime}\right) s_{1}\left(\mathbf{r}^{\prime}\right)\right\} \cdot
$$

\section{Model Tests and Validation}

A detailed validation of this technique will be presented at a later time including experimental and theoretical comparisons. However, in this paper we limit ourselves to 
showing a comparison between the full application of MST and our simplified, faster approach for the coherent component. The incoherent component has been extensively studied in our earlier papers on this subject [7-10].

As in [9], two distinct frequencies, namely $2 \mathrm{GHz}$ and $10 \mathrm{GHz}$, are selected to demonstrate the similarity between the results of the modified PO model and those from MST, with the added significant computation load reduction.

A terrestrial path, elevation of $0^{\circ}$ was selected for illustration. The canopy is assumed to have a cylindrical shape with radius $R=1 \mathrm{~m}$ and height $H=2 \mathrm{~m}$. Vertical polarization is used. A straight receive antenna path, perpendicular to the radio path from the transmitter to the tree, was chosen with part of it in LOS and part shadowed by the tree.

The same material parameters, sizes and orientations of the branches and leaves as in [1], [9], [11] and [12] were considered, yielding effective propagation constants $K_{2 \mathrm{GHz}}=42.06-0.11 j$ and $K_{10 \mathrm{GHz}}=210.34-0.25 j$, respectively, and specific attenuations $\alpha_{\text {spec, } 2 \mathrm{GHz}}=0.96 \mathrm{~dB} / \mathrm{m}$ and $\alpha_{\text {spec, } 10 \mathrm{GHz}}=2.17 \mathrm{~dB} / \mathrm{m}$.

In Fig. 6, we can observe the obtained results: as the traveled distance increases, the resulting field smoothly approaches the incident plane wave field of amplitude $E_{0}=0 \mathrm{dBV} / \mathrm{m}$. Also, as expected, there is a transition region when the path enters the LOS conditions. The overall attenuation behind the canopy at $2 \mathrm{GHz}$ and $10 \mathrm{GHz}$ is


Fig. 6. Total field in test case using MST, and hybrid model with two $K$ approximations. about $2 \mathrm{~dB}$ and $4 \mathrm{~dB}$, respectively. This corresponds to the maximum path traveled through the canopy, which is $2 \mathrm{~m}$, multiplied by $\alpha_{\text {spec, } 2 \mathrm{GHz}}$ or $\alpha_{\text {spec, } 10 \mathrm{GHz}}$.

Had we applied an even simpler model taking into account the geometrical path through the canopy multiplied by the specific attenuation, we would have had a sharp discontinuity in the incident shadow boundary, whereas with MST and our simplified model we get a gentle transition region and not an abrupt one.

The reader is reminded that the specific attenuations are computed using MST. We can also see that it is important to use the phase term in the effective propagation constant, also derived from MST, as depicted in Fig. 5.

Many other theoretical tests and comparisons with measurements were performed proving the validity of our approach [16].

\section{Time-Series Synthesis}

The model presented in this paper can directly be used within time-series synthesizers required for testing overall transmission system behavior. A reasonable assumption is to suppose that the received signal can be modeled, at least over short traveled distances, by means of a Rice model, that is, the sum of a coherent component and a diffuse component.

If, for example, the mobile terminal is traveling in a tree sided road, the received signal will be a mixture of the direct signal, sometimes attenuated by a tree canopy, and multiple contributions from the various trees on either side of the road.

Here is where the model discussed above comes in: we can represent the direct signal with the simplified approach plus a diffuse component. The latter we can actually break down into contributions coming from different parts of the same canopy or from parts of other trees. Since we are dealing with a diffuse component, characterized in terms of power, we can break down each tree into any number of individual volumetric components (voxels). The only condition being that the total average power remains the same regardless of the discretization step. MST provides us with volumetric radar cross section values, $\sigma$, in $\mathrm{m}^{2} / \mathrm{m}^{3}$. The level of discretization is mainly dependent on two points, one is that for the bistatic radar equation to be valid, we need to be in the far field of the target element. We will need to change the size of the discrete voxels if the terminal antenna is too close to the tree. A second criterion is the delay or angle of arrival resolution we require in our application: rougher descriptions would involve coarser steps.

The overall received signal at each route sampling point would be the coherent sum of the direct signal plus all diffuse components supplied with an initial random phase and a changing phase due to the changing distances as the terminal moves. 
The way voxels are generated has been subjected to many hypotheses. We can cite here, for example, the method proposed in [26] to reproduce the non-stationarity in the received signal. In the case of stationary terminals, time variations will still exist due to wind effects. A mechanical method was proposed in [27] which is compatible with our discretization of the tree canopies for describing the diffuse component.

\section{Summary}

In this paper, we have reviewed the application of Multiple Scattering Theory, MST, to the modeling radio link conditions. This technique has usually been utilized in remote sensing. The only main shortcoming we face when applying it to communications or navigation links is the very long computation time required which involve volume integration and averaging over individual component orientations (branches and leaves). However, this technique provides a fast method of calculating tree diffuse scattering. Thus, we propose to replace the computationally inefficient way of calculating the coherent component with a simpler hybrid method involving two paths, one through the canopy and another around the canopy, with the application of an effective propagation constant $K$ derived by MST. We have shown tests where the obtained results are very close also to the experimental datasets and, further, we have outlined the application of the presented model within a time-series generator.

\section{Acknowledgments}

This work was supported by the European Association on Antennas and Propagation (EurAAP).

\section{References}

[1] ITU. Attenuation in Vegetation, ITU-R Rec. P.833-8. Geneva (Switzerland): ITU, 2013.

[2] FOLDY, L. L. The multiple scattering of waves. I. General theory of isotropic scattering by randomly distributed scatterers. Physical Review, 1945, vol. 67, no. 3/4, p. 107-119. DOI: 10.1103/PhysRev.67.107

[3] LAX, M. Multiple scattering of waves. Review of Modern Physics, 1951, vol. 23, no. 4, p. 287-310. DOI: 10.1103/RevModPhys.23.287

[4] TWERSKY, V. Multiple scattering of electromagnetic waves by arbitrary configurations. Journal of Mathematical Physics, 1967, vol. 8, no. 3, p. 589-610. DOI: 10.1063/1.1705237

[5] ISHIMARU, A. Wave Propagation and Scattering in Random Media. Wiley-IEEE Press, 1999. ISBN: 978-0-7803-4717-5

[6] UlABY, F. T., LONG., D. Microwave Radar and Radiometric Remote Sensing. University of Michigan Press, 2014. ISBN-13: 978-0472119356

[7] CHEFFENA, M., PEREZ-FONTAN, F. Land mobile satellite channel simulator along roadside trees. IEEE Antennas and
Wireless Propagation Letters, 2010, vol. 9, p. 748-751. DOI: 10.1109/LAWP.2010.2060465

[8] CHEFFEnA, M., PEREZ-FOnTAN, F., LACOSTE, F., et al. Land mobile satellite dual polarized MIMO channel along roadside trees: modeling and performance evaluation. IEEE Transactions on Antennas and Propagation, 2012, vol. 60, no. 2, p. 597-605. DOI: 10.1109/TAP.2011.2173447

[9] KVICERA, M., ISRAEL, J., PEREZ-FONTAN, F., PECHAC, P. Sensitivity analysis of multiple scattering theory applied to tree canopies at microwave frequencies. IEEE Antennas and Wireless Propagation Letters, 2015, vol. 15, p. 1175-1178. DOI: 10.1109/LAWP.2015.2498645

[10] KVICERA, M., PEREZ-FONTAN, F., PECHAC, P. Scattering from single isolated tree based on physical optics: preliminary model. In Proceedings of the 9th European Conference on Antennas and Propagation (EuCAP). Lisbon (Portugal), 2015.

[11] DE JONG, Y. L. C., HERBEN, M. H. A. J. A tree-scattering model for improved propagation prediction in urban microcells. IEEE Transactions on Vehicular Technology, 2004, vol. 53, no. 2, p. 503-513. DOI: 10.1109/TVT.2004.823493

[12] DE JONG, Y. L. C. Measurement and Modeling of Radiowave Propagation in Urban Microcells. PhD Thesis. T.U. Eindhoven, 2001.

[13] ISRAEL, J., PAJOT, A. Fading and scattering due to trees in L to $\mathrm{Ka}$ band propagation simulations. In Proceedings of the 9th European Conference on Antennas and Propagation (EuCAP). Lisbon (Portugal), 2015.

[14] TORRICO, S. A., BERTONI, H. L., LANG, R. H. Modeling tree effects on path loss in a residential environment. IEEE Transactions on Antennas and Propagation, 1998, vol. 46, no. 6, p. 872-880. DOI: $10.1109 / 8.686776$

[15] CHEE, K. L., TORRICO, S. A., KURNER, T. Radiowave propagation prediction in vegetated residential environments. IEEE Transactions on Vehicular Technology, 2013, vol. 62, no. 2, p. 486-499. DOI: 10.1109/TVT.2012.2226764

[16] KVICERA, M., PEREZ-FONTAN, F., ISRAEL, J., PECHAC, P. Modeling scattering from tree canopies for UAV scenarios. In Proceedings of the 10th European Conference on Antennas and Propagation (EuCAP). Davos (Switzerland), 2016. DOI: 10.1109/EuCAP.2016.7481249

[17] TORRICO, S. A., LANG, R. H. A simplified analytical model to predict the specific attenuation of a tree canopy. IEEE Transactions on Vehicular Technology, 2007, vol. 56, no. 2, p. 696-703. DOI: 10.1109/TVT.2007.891485

[18] KARAM, M. A., FUNG, A. K. Electromagnetic scattering from a layer of finite length, randomly oriented, dielectric, circular cylinders over a rough interface with application to vegetation. International Journal of Remote Sensing, 1988, vol. 9, no. 6, p. 1109-1134. DOI: $10.1080 / 01431168808954918$

[19] KARAM, M. A., FUNG, A. K., ANTAR, Y. M. M. Electromagnetic wave scattering from some vegetation samples. IEEE Transactions on Geoscience and Remote Sensing, 1988, vol. 26, no. 6, p. 799-808. DOI: 10.1109/36.7711

[20] KARAM, M. A., FUNG, A. K. Leaf-shape effects in electromagnetic wave scattering from vegetation. IEEE Transactions on Geoscience and Remote Sensing, 1989, vol. 27, no. 6, p. 687-697. DOI: 10.1109/TGRS.1989.1398241

[21] DIAZ, L., MILligAN, T. Antenna Engineering using Physical Optics: Practical CAD Techniques and Software. Norwood (USA): Artech House, 1996. ISBN:0890067325

[22] BALANIS, C. A. Advanced Engineering Electromagnetics. Wiley, 2012. ISBN : 978-0-470-58948-9

[23] KVICERA, M., PECHAC, P. Seasonal variations of polarization diversity gain in a vegetated area considering high elevation angles 
and a nomadic user. International Journal of Antennas and Propagation, 2015, vol. 2015, 9 p., Article ID 194626. DOI: $10.1155 / 2015 / 194626$

[24] TESCHL F., PEREZ-FONTAN, F., SCHONHUBER, M., et al. Attenuation of spruce, pine, and deciduous woodland at C-band. IEEE Antennas and Wireless Propagation Letters, 2012, vol. 11, p. 109-112. DOI: 10.1109/LAWP.2012.2184253

[25] HORAK, P., PECHAC, P. Excess loss for high elevation angle links shadowed by a single tree: measurements and modelling. IEEE Transactions on Antennas and Propagation, 2012, vol. 60 , no. 7, p. 3541-3545. DOI: 10.1109/TAP.2012.2196944

[26] SCHUBERT, F. M., JAKOBSEN, M. L., FLEURY, B. H. Nonstationary propagation model for scattering volumes with an application to the rural LMS channel. IEEE Transaction on Antennas and Propagation, 2013, vol. 61, no. 5, p. 2817-2828. DOI: 10.1109/TAP.2013.2242821

[27] CHEFFENA, M., EKMAN, T. Dynamic model of signal fading due to swaying vegetation. EURASIP Journal on Wireless Communications and Networking, 2009. DOI: $10.1155 / 2009 / 306876$

\section{About the Authors ...}

Fernando P. FONTÁN was born in Villagarcía de Arosa, Spain. He obtained his degree in Telecommunication Engineering in 1982 from the Technical University of Madrid and his Ph.D. in 1992 from the same university. After working in industry since 1984 he became an Assistant Professor at the University of Vigo in 1988. In 1993 he became an Associate Professor and in 1999 a Full Professor at the Signal Theory and Communications Department of the University of Vigo. He is the author of a number of books and journal papers and has been the leader in several projects funded by public and private entities. He participates in ITU-R WG3 on Propagation Modeling. He has been a Management Committee Member of EU' COST $255,280,297$, and IC0802. His main research interest is in the field of mobile fixed radio communication propagation channel modeling.

Milan KVICERA was born in Mestec Kralove, Czech Republic. He received the M.Sc. degree and the Ph.D. degree in Radio Electronics from the Czech Technical University in Prague, Czech Republic, in 2008 and 2012, respectively. He is currently working as a researcher in the Dept. of Electromagnetic Field, Czech Technical University in Prague. His research interests are focused on radio wave propagation and satellite communications.

Pavel PECHAC (M'94-SM'03) was born in Prague, Czech Republic. He received his M.Sc. degree and Ph.D. degree in Radio Electronics from the Czech Technical University, Prague, Czech Republic, in 1993 and 1999, respectively. He is currently a Professor in the Dept. of Electromagnetic Field, Czech Technical University, Faculty of Electrical Engineering. His research interests are in the field of radio wave propagation and wireless systems. More details can be found at http://pechac.elmag.org.

Jonathan ISRAEL graduated from Télécom ParisTech, Paris, France, with an engineer diploma in Signal Processing and a Master of Science in Mathematics from the Ecole Normale Supérieure of Cachan in 2005. Since 2006, he has been working at ONERA. His fields of interest include physical statistical propagation channel characterization and signal processing for GNSS and satcom applications. 